\title{
IV. Die Kammerwahlen vom April 1928: Bestätigung des Vertrauten
}

Die Kammerwahlen vom 22. und 29. April 1928 fanden wieder nach dem alten, vor dem Ersten Weltkrieg bestehenden Mehrheitswahlrecht statt ${ }^{1}$. Die Aufgabe des komplizierten, erst 1919 eingeführten Mischsystems hatte sich bereits im Vorfeld der Wahlen von 1924 angekündigt, war damals aber noch gescheitert ${ }^{2}$. Die Wahlrechtreform vom Juli 1927 leitete dann die Rückkehr zum vertrauten und nur leicht modifizierten „scrutin d'arrondissement“ ein. Daß die Abgeordnetenkammer bei dieser Entscheidung ihre „âme cartelliste “ wiedergefunden habe, wie Goguel plakativ formuliert hat ${ }^{3}$, ist eine Aussage, die sich allein auf die Mehrheitsverhältnisse bei diesem Votum bezieht ${ }^{4}$. Die Masse der Stimmen für das alte Wahlrecht kam von Sozialisten und Radicaux. Dabei ging es freilich keineswegs um eine Reaktivierung des Kartells, vielmehr spiegelt die Haltung der beiden Fraktionen die seit 1924 erlebten Enttäuschungen und damit auch das Scheitern des linken Parteienbündnisses. Für die SFIO, die eigentlich ein konsequentes Verhältniswahlsystem befürwortete, bedeutete das Mehrheitswahlrecht das kleinere Übel im Vergleich zum Mischsystem mit seinem faktischen Zwang zu überparteilichen Wahllisten. Wurde der Verzicht auf ein klares parteipolitisches Profil bereits 1924 skeptisch gesehen, so war jetzt, nach dem Scheitern des Cartel des Gauches, die Grundlage für gemeinsame Listen mit den Radicaux entfallen. Die Fraktion des Parti radical hatte das alte Wahlrecht ohnehin schon Anfang 1924 wieder einführen wollen. Die Konfusion, die seit Bildung der Union nationale innerhalb der Partei herrschte, war ein zusätzliches Argument für die traditionelle Personalisierung der Wahlentscheidung auf der Ebene des Arrondissements. Innerhalb der Regierung war der radikale Innenminister Sarraut die treibende Kraft für eine Wahlrechtsänderung. Für das „scrutin d'arrondissement“ - „dont la démocratie française a l'habitude et le goût" 5 - sprach aus seiner Sicht vor allem die dadurch errichtete Barriere gegen das weitere Erstarken der Kommunisten. Die entschiedensten Gegner einer Änderung stammten aus den Reihen der konservativen Fédération républicaine, wo die Rückkehr zu den „mares stagnantes“ des alten Wahlrechts mit großer Enttäuschung gesehen wurde ${ }^{6}$.

In der Praxis der Wahlen von 1928, die erneut eine Rekordbeteiligung aufwiesen7, trug das "neue“ alte Wahlrecht im entscheidenden zweiten Wahlgang zur weiteren Auflösung der politischen Fronten bei. Obwohl die große Mehrheit der radikalen Kammerfraktion in den zurückliegenden beiden Jahren die Regierung Poincaré unterstützt hatte und obwohl die radikale Parteiführung an diesem Kurs festhalten wollte, kam es nun in den Wahlbezirken wieder häufig zur traditionel-

1 Vgl. zum folgenden v.a. Huard, Suffrage, S. 232 f.; Bonnefous, Histoire 4, S. 221-227; Mayeur, Vie politique, S. 286-288.

2 Vgl. oben S. $377 \mathrm{f}$.

3 Goguel, Politique, S. 244.

4 Am 12. 7. 1927 wurde die Reform mit 320 zu 234 Stimmen verabschiedet. Vgl. zum gesamten Vorgang Bonnefous, Histoire 4, S. 223-227.

5 So bei Einbringung der Gesetzesvorlage in der Kammer am 10.3. 1927 nach ebd., S. 223.

6 Vgl. Bernard, La dérive des modérés, S. $319 \mathrm{f}$.

7 So wurde im ersten Wahlgang eine Beteiligung von $83,8 \%$ erreicht. Mayeur, Vie politique, S. 287. 
len wechselseitigen Unterstützung zwischen Radicaux und Sozialisten, indem der eigene, im ersten Wahlgang schlechter positionierte Kandidat zurückgezogen wurde. Diese paradoxe Wiederbelebung eines linken Wahlkartells erfuhr noch eine zukunftsträchtige Erweiterung, da die Sozialisten ein derartiges „désistement" teilweise auch gegenüber besser plazierten Kommunisten praktizierten8. Umgekehrt gab es im Zeichen der Union nationale aber auch wechselseitige Unterstützungen von Parti radical und Mitte-rechts-Kandidaten zu Lasten der Sozialisten'. Die einzige Kraft, die sich im zweiten Wahlgang nicht zum taktischen Rückzug bereit erklärte, waren die Kommunisten, die sich der traditionellen Gauche-contre-droite-Strategie entzogen und eine strikte Classe-contre-classeKonzeption verfolgten, nach der auch die SFIO zum bürgerlichen Lager zählte ${ }^{10}$.

Im Gesamtergebnis ließen sich die Wahlen von 1928 als klares Plebiszit für die seit Mitte 1926 von Poincaré verfolgte Politik der Union nationale und insbesondere auch als breite Anerkennung der faktischen Währungsstabilisierung deuten. Le Temps stellte daher nach dem zweiten Wahlgang mit Recht fest: „La qualité essentielle des élections, c'est le désir manifesté par le pays de voir continuer la politique inaugurée et suivie par M. Poincaré depuis qu'il a repris le pouvoir le 23 juillet 1926." 11 Gestärkt wurde vor allem die politische Heimat des Ministerpräsidenten, d. h. die Kräfte des rechten Liberalismus im Umfeld der Alliance démocratique, die nach Neuformierung der Kammerfraktionen nun über 120 Abgeordnete stellten (1924 etwa 80) ${ }^{12}$. Die konservative Union républicaine démocratique hielt ihre Position von gut 100 Mandaten, was angesichts der erhöhten Gesamtzahl der Kammerabgeordneten einer leichten Schwächung gleichkam. Der Parti radical mußte einen kleinen Verlust hinnehmen (125 statt 139 Mandate). Nimmt man alle nominellen Fraktionen des Regierungslagers zusammen, so erreichte dieses jetzt eine Stärke von über 450 Abgeordneten, das sind etwa $75 \%$ aller Deputierten gegenüber zuletzt etwa 370 (64\%). Innerhalb der Union nationale hatten sich die Gewichte jetzt so verschoben, daß die Unterstützung des Parti radical zur Mehrheitsbildung nicht mehr unbedingt nötig war ${ }^{13}$. Eine wichtige Voraussetzung des breiten Regierungsbündnisses war damit gerade durch den Erfolg Poincarés aufgehoben worden. Nahezu unverändert blieb die Zahl der sozialistischen Mandate (100 gegenüber 104 nach den Wahlen von 1924), während die Kommunisten - trotz erhöhter Stimmenzahl im ersten Wahlgang ${ }^{14}$ - einen deutlichen Rückgang (11 gegenüber 26) verzeichnen mußten.

Das Wahlergebnis von 1928 und vor allem das in der Mandatszahl schwache Abschneiden der Kommunisten bedeuteten auch - und darin stand es im Gegensatz zu den die deutsche Inflationskrise "quittierenden" Ergebnissen der beiden

8 Vgl. Ziebura, Blum, S. 386 f.

9 Teilweise gab es sogar Rücktritte von Kandidaten der Fédération républicaine zugunsten von Radicaux. Vgl. Bernard, La dérive des modérés, S. 318.

10 Vgl. Fauvet, Histoire, S. 74-77; Wirsching, Vom Weltkrieg zum Bürgerkrieg?, S. $396 \mathrm{f}$.

$"$ LT, 2. 5. 1928, S. 1, „Tout pour union“.

12 Zu den absoluten Zahlen im ersten Wahlgang vgl. Mayeur, Vie politique, S. 287.

13 Die bisherigen "Regierungsfraktionen" erreichten ohne Radicaux etwa 325 von 610 Mandate, das sind etwa $53 \%$. Fraglich war auch die Haltung der 18 Républicains-socialistes.

14 Etwa 1 Million Stimmen gegenüber rund $875000 \mathrm{im}$ Mai 1924. Nach Levêque, Histoire des forces politiques, S. 174-179. 
Reichstagswahlen von 1924 - eine Stärkung der systemloyalen Kräfte in der Abgeordnetenkammer. Daß hierfür die Wahlrechtsänderung eine wesentliche Rolle spielte, steht außer Zweifel. Im Vergleich zu den großen SPD-Verlusten von 1924 sei allerdings auch betont, daß die SFIO das Experiment einer sozialistischen Beteiligung am Regierungslager trotz ihres blassen Profils innerhalb des Cartel des Gauches gut überstanden hat. Nach absoluten Stimmen erreichte die Partei nahezu das Niveau der Kammerwahlen von 1919 - d.h. vor der Spaltung von Tours! -, und erstmals in ihrer Geschichte konnte sie jetzt im ersten Wahlgang die Stimmenzahl des Parti radical übertreffen ${ }^{15}$. Der gewachsene kommunistische Wähleranteil ging also, wenngleich es regional teils bedeutende Verschiebungen gab, nicht zu Lasten der Sozialisten. Die vorsichtigen Ansätze zu einer Koalitionspolitik hatten demnach keine besonders negativen wahlpolitischen Konsequenzen. Zudem war es der SFIO seit Mitte 1926 wohl auch in den Augen zahlreicher Wähler gelungen, wieder überzeugend die Rolle einer loyalen Opposition einzunehmen. Dies half der Partei, vor allem in ländlichen Regionen Stimmen aus dem ursprünglich radikalen Wählerreservoir an sich ziehen und in den Industriegebieten eine stärkere Abwanderung zu den Kommunisten verhindern'16. Allerdings wurde das relativ gute sozialistische Ergebnis durch die persönliche Niederlage Blums gegen den Kommunisten Jacques Duclos in einem Pariser Wahlbezirk etwas überschattet ${ }^{17}$.

Während die Maiwahlen von 1924 den Aufbruch in eine vage linke Hoffnung markiert hatten, siegte bei den Kammerwahlen vom April 1928 in mehrfacher Hinsicht das Vertraute. Bestätigt wurden in erster Linie der erfolgreiche Ministerpräsident Poincaré, sein Konzept einer breiten nationalen Kooperation der politischen Kräfte rechts von den Sozialisten und seine sozialkonservative, primär auf indirekte Steuern setzende Finanzpolitik. Bestätigt wurde damit aber auch Poincarés Verständnis des parlamentarischen Regierungssystems, das nicht auf ein Blockdenken und Parteienbündnisse setzte, sondern auf die überparteiliche Führung durch einen starken Président $d u$ conseil. In einem weiteren Sinn aber wurden alle systemloyalen Kräfte der Abgeordnetenkammer gestärkt. Die Dritte Französische Republik schien ihre Krise der Inflationszeit bestens überstanden zu haben.

Angesichts des Wahlergebnisses gab es zunächst keinen Grund für einen Regierungswechsel. Das durch den Vertrauensbeweis legitimierte Kabinett Poincaré IV blieb weiterhin im Amt und machte sich nun daran, das Werk der Währungsstabilisierung durch die inzwischen auch weithin akzeptierte formelle Abwertung abzuschließen. Der französische Franc wurde am 25. Juni 1928 mit einem Dollar-

15 Die SFIO erreichte etwa 1,7 Millionen Stimmen gegenüber 1919 1,73 Millionen. (Beide Zahlen sind insofern vergleichbar, weil sich die SFIO 1919 an keiner Listenverbindung beteiligt hat.) Der Parti radical kam 1928 auf etwa 1,68 Millionen. Zahlen nach Mayeur, Vie politique, S. 256 und 287. Vgl. auch Ziebura, Blum, S. 387 f.

16 Differenzierte Analyse in Lefranc, Mouvement socialiste 2, S. 275-278. Der SFIO kam zudem die in Arbeiterkreisen unpopuläre „Klasse-gegen-Klasse“-Strategie der Kommunisten sowie ein konjunktureller Aufschwung mit Lohnverbesserungen zugute. Ein größerer kommunistischer Erfolg wurde auch durch die 1927 erfolgten Richtungskämpfe und den dabei erfolgten Ausschluß der Trotzkisten verhindert. Vgl. Wirsching, Vom Weltkrieg zum Bürgerkrieg?, S. $395 \mathrm{f}$.

17 Im 20. Pariser Arrondissement. Vgl. Ziebura, Blum, S. 385-387. 
preis von 25,53 fixiert, d. h. auf jenem Niveau, das bereits Ende 1926 erreicht worden war ${ }^{18}$. Der neue „franc Poincaréc, der wieder zum Goldstandard zurückkehrte ${ }^{19}$, entsprach damit etwa einem Fünftel des „franc Germinal“ der Vorkriegszeit. Das dazu nötige Gesetz war einen Tag zuvor in der Abgeordnetenkammer mit einer geradezu überwältigenden Mehrheit von 448 zu 18 Stimmen bei 133 Enthaltungen verabschiedet worden ${ }^{20}$. Die Gegenstimmen kamen im wesentlichen von den Kommunisten, die Enthaltungen von der SFIO, die im Prinzip aber mit der Abwertung völlig einverstanden war. Noch klarer war die Zustimmung im Senat ${ }^{21}$. Der parlamentarische Schlußpunkt unter die Wirren der Inflationszeit erfolgte so in weitgehender politischer Einigkeit.

Nachdem die Union nationale unter Einschluß der Radicaux infolge der Kammerwahlen ihre Funktion der parlamentarischen Mehrheitsbeschaffung verloren hatte, entfiel nun seit der Abwertung des Franc auch ihre Aufgabe als breites Stabilisierungsbündnis. Mit dem folgerichtigen Regierungsaustritt des Parti radical im November $1928^{22}$ wurde Poincarés Modell des überparteilichen Ministerpräsidenten von den veränderten politischen Realitäten eingeholt, und mit dem krankheitsbedingten Rücktritt des erfolgreichen Regierungschefs im Sommer 192923 begann eine neue Periode der parlamentarischen Instabilität und der raschen $\mathrm{Ab}-$ folge von Kabinetten, wie es dem klassischen Muster des französischen Parlamentarismus entsprach.

18 Vgl. zur Abwertung Fohlen, Frankreich, S. 108; Sauvy, Histoire économique 1, S. 95 f.; Blancheton, Le Pape et l'Empereur, S. 412-414.

19 Im Kontext der Ursachenanalyse für die Weltwirtschaftskrise wird die insbesondere die französische Rückkehr zum Goldstandard wirtschaftshistorisch überwiegend kritisch diskutiert. Vgl. aus der neuesten Literatur v.a. Eichengreen, Golden Fetters, und Mouré, The Gold Standard Illusion. Ebd., S. 180-220, sowie ders., Managing the Franc Poincaré, zur weiteren währungspolitischen Entwicklung in Frankreich.

20 Vgl. Bonnefous, Histoire 4, S. 266-268. Liste der namentlichen Abstimmung in JO, Débats, Chambre 1928, S. 2078 f.

21 Mit 256 zu 3 Stimmen. Bonnefous, Histoire 4, S. 268.

22 Vgl. Berstein, Histoire 2, S. 127-131.

23 Fünf Jahre später, am 15. 10. 1934, verstarb Poincaré im Alter von 74 Jahren. Zum Rücktritt und zum Lebensende vgl. Roth, Poincaré, S. 541-558. 